\title{
Osteossíntese a fio de aço do ângulo mandibular e bloqueio maxilo-mandibular: relato de caso
}

Mandibular angle steel wire osteosynthesis and maxillo-mandibular blocking: case report Osteosíntesis de hilo de acero de ángulo mandibular y bloqueo maxilo-mandibular: reporte de caso

Carla Beatriz Fernandes de OLIVEIRA ${ }^{\mathbf{1}}$ Karolina Braga COSTA ${ }^{1}$

Maísa de Oliveira AGUILLERA ${ }^{2}$

José Carlos Garcia de MENDONÇA ${ }^{3}$ Gustavo Silva PELISSARO 4

Janayna Gomes PAIVA-OLIVEIRA 4

Julio Cesar Leite da SILVA ${ }^{5}$

Ellen Cristina GAETTI-JARDIM ${ }^{5}$

${ }^{1}$ Graduanda em Odontologia da Faculdade de Odontologia da Universidade Federal de Mato Grosso do Sul (Faodo-UFMS), membro da Liga Acadêmica de Cirurgia e Traumatologia Bucomaxilofacial (LACTBMF) da Faodo-UFMS

${ }^{2}$ Residente em Cirurgia e Traumatologia Buco-maxilo-facial da Faodo-UFMS. Coordenadora Pós-graduanda da LACTBMF

${ }^{3}$ Docente da Graduação em Odontologia da Faodo-UFMS, Coordenador do Curso de Residência em Cirurgia e Traumatologia Bucomaxilofacial da Faodo-UFMS e docente da LACTBMF

${ }^{4}$ Preceptor(a) da Residência em Cirurgia e Traumatologia Bucomaxilofacial da Faodo-UFMS e da LACTBMF

${ }^{5}$ Docente da Graduação em Odontologia da Faodo-UFMS, Preceptor(a) da Residência em Cirurgia e Traumatologia Bucomaxilofacial da Faodo-UFMS e da LACTBMF

\section{Resumo}

A mandíbula é um dos ossos mais acometidos pelo trauma facial em virtude de sua projeção anteriorizada favorecendo o acometimento de fraturas. A localização das fraturas se relaciona às áreas anatômicas de resistência e fragilidade por onde a força do impacto será distribuída. O tratamento baseia-se na redução e fixação dos fragmentos ósseos, para isso existe diversas técnicas descritas na literatura, dentre elas, a combinação de fixação intermaxilar (FIM) e osteossíntese com fio de aço. O objetivo deste trabalho é relatar um caso clínico de paciente com fratura em ângulo de mandíbula, submetido a osteossíntese com fio de aço e FIM. Relato do caso: Paciente, 24 anos, gênero masculino, compareceu ao Hospital Maria Aparecida Pedrossian com histórico de queda de bicicleta há quinze dias com trauma na região de mandíbula esquerda cursando com dificuldade de abertura bucal, dor, apresentando ao exame tomográfico fratura de ângulo da mandíbula no lado esquerdo. Diante do exame clinico e tomográfico, optou-se como conduta terapêutica a FIM e o tratamento cirúrgico sob anestesia geral para osteossíntese com fio de aço, que se mostrou uma técnica alternativa a fixação interna rígida com resultados satisfatórios.

Descritores: Fraturas Ósseas; Mandíbula; Fixação de Fratura.

\section{Abstract}

The mandible is one of the bones most affected by facial trauma due to its anterior projection favoring the involvement of fractures. The location of the fractures is related to the anatomical areas of resistance and fragility where the force of the impact will be distributed. The treatment is based on the reduction and fixation of bone fragments, for this there are several techniques described in the literature, among them, the combination of intermaxillary fixation (FIM) and osteosynthesis with steel wire. The objective of this work is to report a clinical case of a patient with a mandible angle fracture, submitted to osteosynthesis with steel wire and FIM. Case report: Patient, 24 years old, male, attended the Hospital Maria Aparecida Pedrossian with a history of falling from a bicycle for fifteen days with trauma in the region of the left jaw, with difficulty in mouth opening, pain, presenting an angle fracture on tomography. of the jaw on the left side. In view of the clinical and tomographic examination, FIM and surgical treatment under general anesthesia for osteosynthesis with steel wire were chosen as a therapeutic approach, which proved to be an alternative technique to rigid internal fixation with satisfactory results.

Descriptors: Fractures, Bone; Mandible; Fracture Fixation.

\section{Resumen}

La mandíbula es uno de los huesos más afectados por el traumatismo facial por su proyección anterior favoreciendo la afectación de fracturas. La ubicación de las fracturas está relacionada con las áreas anatómicas de resistencia Y fragilidad donde se distribuirá la fuerza del impacto. El tratamiento se basa en la reducción Y fijación de fragmentos óseos, para ello existen varias técnicas descritas en la literatura, entre ellas, la combinación de fijación intermaxilar (FIM) Y osteosíntesis con alambre de acero. El objetivo de este trabajo es reportar un caso clínico de un paciente con fractura del ángulo de mandíbula, sometido a osteosíntesis con alambre de acero Y FIM. Caso clínico: Paciente de 24 años, varón, que acudió al Hospital María Aparecida Pedrossian con antecedente de caída de bicicleta durante quince días con traumatismo en la región de la mandíbula izquierda, con dificultad para abrir la boca, dolor, presentando una fractura de ángulo en la tomografía. de la mandíbula del lado izquierdo. Ante el examen clínico $Y$ tomográfico se eligió como abordaje terapéutico la FIM Y el tratamiento quirúrgico bajo anestesia general para la osteosíntesis con alambre de acero, que resultó ser una técnica alternativa a la fijación interna rígida con resultados satisfactorios.

Descriptores: Fracturas Óseas; Mandíbula; Fijación de Fractura.

INTRODUÇÃO

A mandíbula é um dos ossos mais acometidos pelo trauma facial em virtude de sua projeção anteriorizada favorecendo o acometimento de fraturas ${ }^{1,2}$. A localização das fraturas se relaciona às áreas anatômicas de resistência e fragilidade por onde a força do impacto será distribuída sendo as de corpo, ângulo e côndilo mandibulares as mais frequentes com 29,5\%, $27,3 \%$ e $21,1 \%$, respectivamente $^{3}$. Entre as principais causas estão os acidentes de trânsito, as agressões físicas, as quedas e as lesões desportivas sobretudo no sexo masculino ${ }^{4,5}$.

Para a adequada reparação tecidual, as fraturas mandibulares necessitam de estabilização óssea adequada e restauração da oclusão, principalmente as fraturas com deslocamento ${ }^{6}$. Há uma variedade de técnicas 
descritas na literatura para o manejo, dentre elas, fixação intermaxilar (FIM), combinação de FIM e osteossíntese com fio de aço, utilização de parafusos pela técnica lag screw e a fixação com placas e parafusos de titânio, sendo estes últimos tidos como o padrão ouro".

A técnica de redução aberta com osteossíntese utilizando fio de aço é classificada como fixação interna não rígida, ou seja, ocorre movimento entre os fragmentos ósseos ao longo da linha de fratura no momento da mastigação ou mesmo fonação, desencadeando uma cicatrização óssea indireta. Os fios de aço não são suficientemente estáveis para possibilitar o uso da mandíbula durante o processo de cicatrização, por conta disso a fixação maxilo-mandibular é aplicada para manter a estabilidade no período de recuperação pós-operatória ${ }^{3}$. O uso do fio de aço e o bloqueio maxilo-mandibular no tratamento das fraturas mandibulares é um método altamente eficaz quando bem indicado, porém a característica maleável do fio, em alguns casos impossibilita a manutenção dos segmentos fraturados em posição ${ }^{7}$.

O objetivo deste trabalho é relatar um caso clínico de paciente com fratura em ângulo de mandíbula, submetido à osteossíntese com fio de aço e fixação intermaxilar, evidenciando que apesar dos avanços em tecnologia, podemos lançar mão de materiais de custo acessível quando necessário no âmbito de saúde pública, obtendo resultados satisfatórios no tratamento.

\section{CASO CLÍNICO}

Paciente do sexo masculino, 24 anos, compareceu ao serviço de Cirurgia e Traumatologia Bucomaxilofacial do Hospital Universitário Maria Aparecida com histórico de queda de bicicleta há quinze dias com trauma na região de mandíbula esquerda cursando com dificuldade de abertura bucal e dor. Ao exame físico extraoral, apresentou edema importante em região de ângulo mandibular esquerdo, queixas álgicas à palpação na mesma região e trismo e processo infeccioso local, sendo impossibilitado exame clínico intraoral no primeiro momento. A imagem de tomografia computadorizada revelou fratura de ângulo da mandíbula no lado esquerdo (Figura 1A a 1C). Diante do quadro infeccioso instalado, paciente foi internado para tratamento com antibioticoterapia e drenagem de abscesso sob anestesia local. Após melhora de abertura bucal foi realizado a redução incruenta da fratura, instalação de barra de Erich e bloqueio intermaxilar em ambiente ambulatorial. Após cinco dias de internação, paciente apresentou remissão da infecção, e diante de uma oclusão estável e alinhamento dos fragmentos fraturados, optou-se por tratamento cirúrgico com osteossíntese de fio de aço uma vez que o serviço hospitalar no momento não contava com placas e parafusos de titânio.
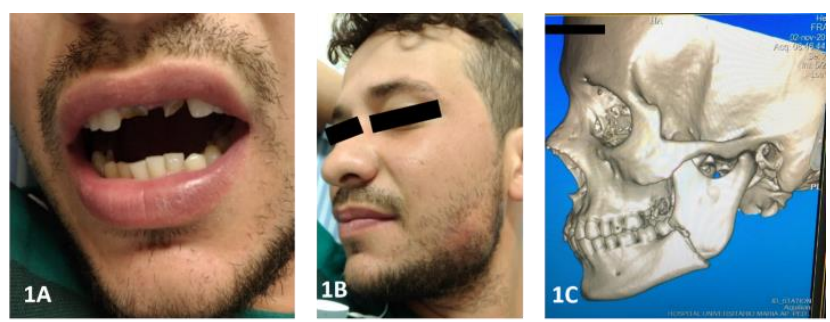

Figuras 1A a 1C: Aspectos pré-operatórios. Limitação de abertura bucal e fraturas dentais, abscesso em região de ângulo a esquerda e tomografia denotando fratura de ângulo mandibular esquerdo.

Sob anestesia geral e intubação nasotraqueal procedeu-se a assepsia intra e extraoral com clorexidina $0.12 \%$ e clorexidina alcoólica $0.5 \%$ respectivamente e aposição de campos estéreis. Foram realizadas infiltrações com lidocaína a 2\% com epinefrina 1:100.000 subcutâneo em região submandibular esquerdo. Os segmentos fraturados foram abordados por acesso de Risdon, feita a redução cirúrgica, perfuração dos cotos ósseos fraturados e osteossíntese com fio de aço em forma de "X". Posteriormente, sutura dos planos internos com fio vicryl 4-0 e sutura intradérmica em pele com fio nylon 5-0. Foi mantido o bloqueio maxilomandibular pós-operatório (Figuras 2A a 2D).
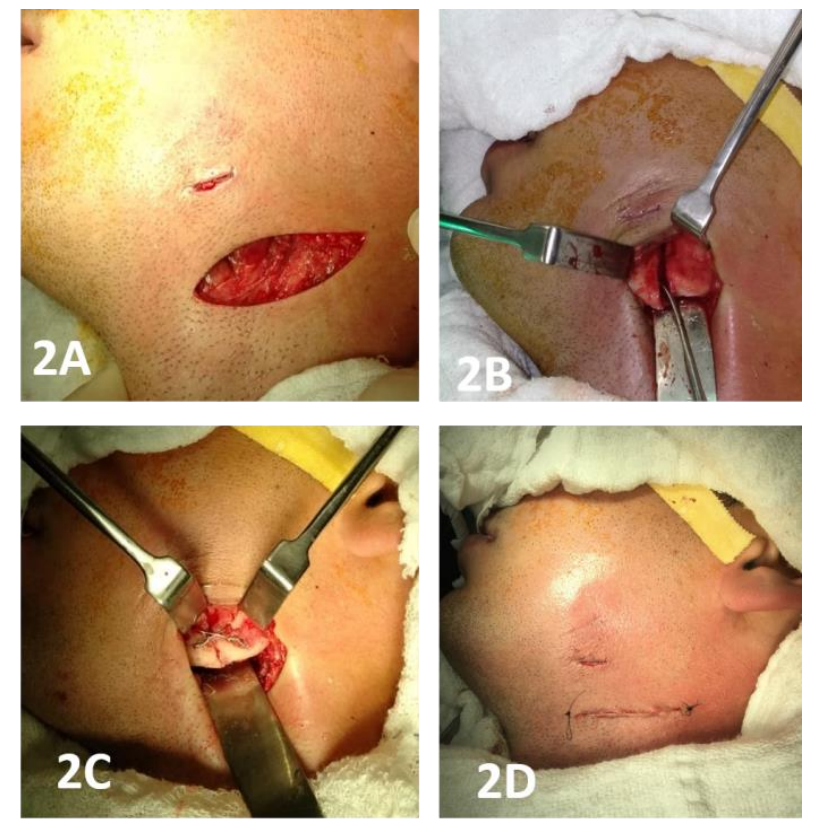

Figuras 2A a 2D: Aspectos trans-operatórios. Acesso, exposição e redução da fratura e sutura intradérmica.

Em pós-operatório imediato, paciente apresentou ao exame clinico, edema em região submandibular esquerda compatível com o procedimento cirúrgico, sem queixas álgicas, e 
redução satisfatória da fratura ao exame tomográfico (Figuras 3A a 3D).
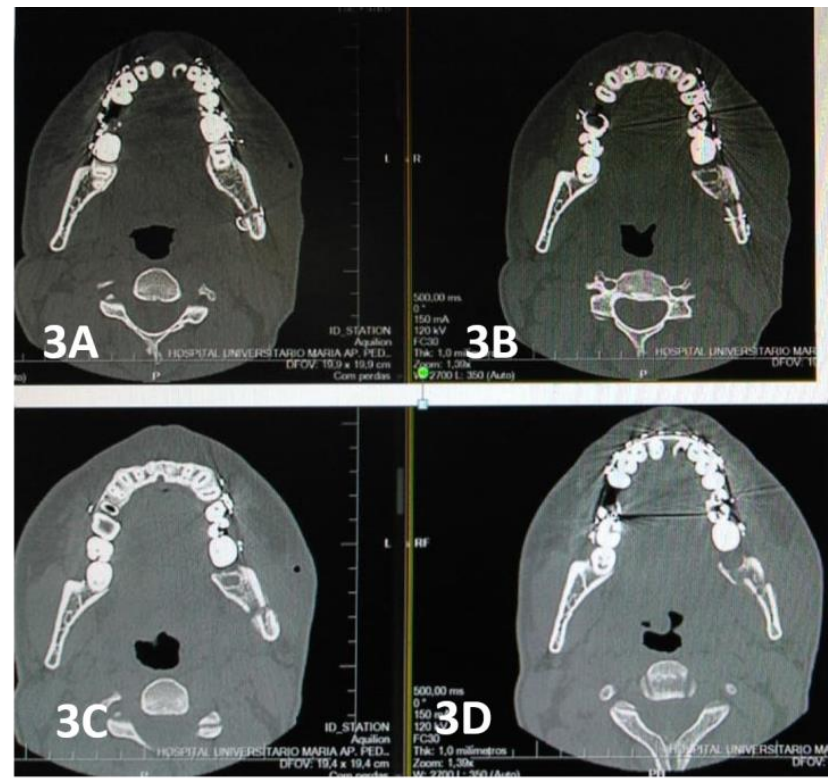

Figuras 3A a 3D: Tomografias computadorizadas em corte axial comparando o alinhamento satisfatório após o procedimento.

Em acompanhamento pós-operatórios de 7 dias, paciente retorna referindo oclusão dentaria sem alterações, apresentando ao exame clínico higiene bucal adequada, bloqueio maxilo-mandibular em posição e uma boa cicatrização da região de acesso cirúrgico, livre de alterações neurosensitivas. Após 45 dias de acompanhamento, foi realizado a remoção do bloqueio e barra de erich sob anestesia local, orientações verbais e por escrito e orientado a necessidade de seguimento de retornos pósoperatórios.

DISCUSSÃO

Podemos ver que historicamente, a fratura do ângulo mandibular necessita de alguma forma de fixação, para controlar a posição do ramo. Existe uma tendência clara em escolher o uso da fixação interna rígida como técnica de primeira eleição, ao uso de materiais como fio de aço e fixação externa tendo em vista que, além de fornecer as vantagens dos outros materiais, eles diminuem ou eliminam o tempo de FIM, aumentando o conforto do paciente no pós-operatório ${ }^{8}$.

Contudo existem desafios na saúde pública, como por exemplo, recursos limitados, a falta de insumos, que precisam ser contornados para assegurar a disponibilidade e acessibilidade à saúde para toda a população. No presente trabalho, oferecemos ao paciente um tratamento alternativo e eficaz na falta de materiais de fixação interna rígida que o serviço não dispunha no momento.

O acesso a região do ângulo da mandíbula pode ser obtido através de incisões transcutâneas ou transmucosas, a fim de expor e reduzir as linhas de fraturas ${ }^{9,10}$. Com base na literatura, o acesso cirúrgico de escolha foi o Acesso de Risdon, através dele a região submandibular é abordada extraoralmente com uma incisão abaixo e atrás do ângulo da mandíbula ${ }^{11}$, fornecendo boa visualização e favorecendo a redução anatômica, além de apresentar baixo índice de contaminação ${ }^{12}$.

Existem vários métodos para a fixação intermaxilar, o escolhido para conduzir o caso foi a Barra de Erich, procedimento descrito por Erich em $1942^{13}$. Esse tipo de bloqueio estabiliza a oclusão para o tratamento da fratura, por conseguinte obtemos um guia para posicionar os fragmentos ósseos ${ }^{14}$. O que é considerado um padrão de cuidado para FIM pois promove estabilidade ${ }^{15}$. A sua escolha para esse caso foi possível porque o paciente possuía número de dentes suficientes e adequado suporte periodontal, permitindo boa ancoragem para as amarrias com fios de aço.

Apesar da literatura relatar desvantagens do uso da Barra de Erich, dentre eles, dano periodontais, extrusão em dentes uniradiculares, perda óssea próximo a barra ${ }^{16}$. Tais aspectos não foram constatados no presente caso, devido a correta execução da técnica na instalação do sistema e por conta do controle de higiene oral realizado pelo paciente.

O fio de aço utilizado na osteossíntese, promoveu a junção dos segmentos fraturados e a FIM forneceu imobilização, assim como, a estabilidade oclusal necessária para a formação do calo ósseo, de forma que a união óssea pode ocorrer.

CONCLUSÃO

Existe uma variedade de técnicas cirúrgicas bem sucedidas descritas na literatura para o tratamento das fraturas do ângulo mandibular, demostrando que não existe apenas um único método eficaz. As habilidades e capacidade do cirurgião para executar corretamente a técnica, juntamente com os prós e contras associados a cada plano de tratamento, podem definir o sucesso dos casos em lugares que não dispõe de tantos recursos o que foi evidenciado no caso apresentado.

\section{AGRADECIMENTOS}

O presente trabalho foi realizado com apoio da Fundação Universidade Federal de Mato Grosso do Sul - UFMS/MEC - Brasil.

\section{REFERÊNCIAS}

1. Ravikumar C, Bhoj M. Evaluation of postoperative complications of open reduction and internal fixation in the management of 
mandibular fractures: A retrospective study. Indian J Dent Res. 2019;30(1):94-6.

2. Morris $\mathrm{C}$, Bebeau NP, Brockhoff $\mathrm{H}$, Tandon R, Tiwana P. Mandibular fractures: An analysis of the epidemiology and patterns of injury in 4,143 fractures. J Oral Maxillofac Surg 2015;73(5):951.e1-951.e12.

3. Miloro M, Waiter PD, Larsen PE, Ghali GE. Princípios de cirurgia bucomaxilofacial de Peterson. 3.ed. São Paulo: Santos; 2016.

4. Motamedi MHK. An assessment of maxillofacial fractures: A 5-year study of 237 patients. J Oral Maxillofac Surg. 2003;61(1):61-4.

5. Ellis E, Moos KF, El-Attar A. Ten years of mandibular fractures: An analysis of 2,137 cases. Oral Surgery, Oral Med Oral Pathol. 1985;59(2):120-29.

6. Hollier LH. Evidence-Based Medicine: Mandible Fractures. 2017;192-200.

7. Franck FC, Oliveira Júnior PA de, Vitale $M$, Pino SD, Dias FJN. Meios de fixação mais utilizados em fraturas de ângulo mandibular. 2014;2(1):25-32.

8. Ellis E. Management of fractures through the angle of the mandible. Oral Maxillofac Surg Clin North Am. 2009;21(2):163-74.

9. Chen CL, Zenga J, Ruchin Patel, Branham G. Complications and Reoperations in Mandibular Angle Fractures. JAMA Facial Plast Surg. 2018;20(3):238-43.

10. Cillo JE, Ellis E. Management of bilateral mandibular angle fractures with combined rigid and nonrigid fixation. J Oral Maxillofac Surg. 2014;72(1):106-11.

11. Kreutziger KL. Surgical anatomy and surgical incisions. Oral Surg. 1984;58(6):637-46.

12. Mendonça JCG De, Quadros DC De, JardimGaetti EC, Santos CM Dos, Masocatto DC, Oliveira MM, et al. Acesso extraoral para ostessíntese de fratura de ângulo mandibular. Arch Heal Invest. 2015;4(6):9-14.

13. Erich JB. Treatment of Fractures of the Upper Jaw. J Am Dent Assoc. 1942;29(5):783-93.

14. Kirk D, Whitney J, Shafer D, Song L. Tight Placement of Erich Arch Bar while Avoiding Wire Fatigue Failure. J Oral Maxillofac Surg. 2016;74(3):562-68.

15. Koshy JC, Feldman EM, Chike-Obi CJ, Bullocks JM. Pearls of mandibular trauma management. Semin Plast Surg. 2010;24(4):357-74.

16. Ferreira AGM, Weismann R, Heitz C, Oliveira MG de, Woitchunas GFP. Três métodos de bloqueio maxilomandibular para o tratamento das fraturas mandibulares. RFO UPF. 2004; 68-72.

\section{CONFLITO DE INTERESSES}

Os autores declaram não haver conflitos de interesse

\section{AUTOR PARA CORRESPONDÊNCIA}

\section{Ellen Cristina Gaetti Jardim}

Universidade Federal de Mato Grosso do Sul

Cidade Universitária

79070-900 Campo Grande - MS, Brasil

+55 (67) 3345-7682

E-mail: ellen.jardim@ufms.br 